\title{
Magnetic resonance spectroscopy as a promising modality for assessing ketogenic diet impact on the level of cerebral metabolites in the treatment of certain neurological disorders
}

\author{
Piotr Artur Machowiec ${ }^{1, A-E \oplus}$, Marcela Maksymowicz ${ }^{1, C-D \oplus}$, Halina Piecewicz-Szczęsna ${ }^{1, C, E-F} \oplus$ \\ ${ }^{1}$ Chair and Department of Epidemiology and Clinical Research Methodology, Medical University, Lublin, Poland \\ A - Research concept and design, B - Collection and/or assembly of data, C - Data analysis and interpretation, \\ $D$ - Writing the article, E-Critical revision of the article, $F$ - Final approval of the article
}

\begin{abstract}
Machowiec PA, Maksymowicz M, Piecewicz-Szczęsna H. Magnetic resonance spectroscopy as a promising modality for assessing ketogenic diet impact on the level of cerebral metabolites in the treatment of certain neurological disorders. Ann Agric Environ Med. 2022; 29(2): 201-206. doi: $10.26444 /$ aaem/145069
\end{abstract}

\begin{abstract}
Introduction and Objective. The ketogenic diet (KD) is a high-fat, adequate-protein, low-carbohydrate diet that is getting more and more widespread in medicine. This dietary intervention causes changes in cerebral metabolism, which are considered potentially beneficial in patients with neurological disorders, but its impact should be controlled and assessed individually. The aim of this review is to provide an update of existing evidence concerning the utility of magnetic resonance spectroscopy (MRS) in monitoring shifts in the cerebral metabolism during ketogenic diet in patients with neurological disorders.

Review Methods. The latest available literature was reviewed by May 13, 2021 using the PubMed, Web of Science and Google Scholar databases. There were 13 papers selected for analysis after reading the title, abstracts and whole text, meeting the assumed criteria.

Abbreviated description of the state of knowledge. MRS is a non-invasive imaging method providing information about the metabolism of brain tissues and playing an increasingly important role in monitoring the concentrations of cerebral metabolites in the course of such neurological disorders as primary brain tumour, epilepsy during KD. Recent trials prove that inverse correlation between serum $\beta$-hydroxybutyrate levels and $\mathrm{N}$-acetylaspartate in brain tissue confirm antiepileptogenic properties of KD. Furthermore, ketone concentrations including $\beta$-hydroxybutyrate and acetone in both lesional and contralateral brain are referred to as correlating with average ketonuria in patients with primary brain tumours. Summary. MRS is a feasible tool for detecting cerebral metabolic shifts linked to a ketogenic diet. However, further studies confirming MR spectroscopy utility in monitoring ketogenic diet treatment in patients with neurological disorders are needed.
\end{abstract}

Key words

magnetic resonance spectroscopy, ketogenic diet, cerebral metabolism

\section{INTRODUCTION AND OBJECTIVE}

Ketogenic diets (KDs) have been very popular for years, mainly in societies facing significant problems of overweight and obesity $[1,2,3]$. A diet high in fat and low in carbohydrates limits the consumption of carbohydrates to $20-50 \mathrm{~g} /$ day, which leads to a decrease in insulin secretion and enables switching to fat burning imitating the state of fasting [1]. Consequently, it contributes to the conversion of fatty acids into ketone bodies.

Furthermore, it is also getting more and more successfully used in medicine, especially in patients with neurologic disorders. KD is considered to be an effective and safe therapy in children and adolescents with refractory epilepsy $[4,5,6]$. Besides, attempts to use KD in adult patients with epilepsy seem to be promising [7]. It may also exert neuroprotective impact in cases of brain damage and Alzheimer's or Parkinson's diseases [8]. Another area of use of this diet

Address for correspondence: Piotr Artur Machowiec, Chair and Department of Epidemiology and Clinical Research Methodology, Medical University, Lublin, Poland

E-mail: piotr.machowiec1997@gmail.com

Received: 03.08.2021; accepted: 17.12.2021; first published: 28.12.2021 is in adjunctive cancer therapy. $\mathrm{KD}$ seems to be feasible in inducing ketosis in heavily pretreated patients with recurrent glioma [9]. There are also reports of its impact on physical function, perceived energy, and food cravings in women with ovarian and endometrial cancer [10] as well as on exploiting glycolytic nature of cancer cells improving body composition and lowering serum insulin [11]. Moreover, it may play a significant role in adjuvant treatment of breast cancer, influencing body composition, blood parameters and survival of patients with beneficial effects [12]. The preclinical experiments demonstrated that $\mathrm{KD}$ increased radiation sensitivity in case of pancreatic cancer [13].

Nevertheless, due to the fact that KDs are deficient in a number of valuable nutrients, their common and chronic use is sometimes referred to as a disturbing and poorly understood phenomenon [8]. However, KD in many neurological disorders is a recognized and effective method of treatment. It is underlined that its mechanisms of action in the brain seem to be omni-directional, so it prompts to evaluate the functioning of the brain under pathological conditions in relation to the normal brain during $\mathrm{KD}$ therapy [8]. That's why this highlights the importance of magnetic resonance spectroscopy (MRS) in monitoring the ketogenic 
diet's impact on certain metabolites' level in the brain and assessing the response to such adjuvant diet treatment in patients with neurological disorders.

The aim of this review is to provide an update of existing evidence concerning the utility of MRS in evaluating KD impact on shifts in the cerebral metabolism in patients with neurological disorders.

\section{REVIEW METHODS}

This review was guided by the PRISMA statement and has the highest level of evidence compared to other study methods. The search was undertaken on 13 May 2021. To find articles, the following keywords were used: "MRS" OR "magnetic resonance spectroscopy" AND "ketogenic diet" OR "high-fat diet" AND "cerebral metabolism" OR "neurological disorders". Sources derived from PubMed, Web of Science and Google Scholar databases, meeting the inclusion criteria summarized in the table (Tab. 1) and delving into KD and role of MRS in detecting cerebral metabolism shifts associated with this type of diet. According to the PICOS ( $\mathrm{P}$ - population - Participants, I -Intervention, $\mathrm{C}$ Comparison, $\mathrm{O}$ - Outcomes, $\mathrm{S}$ - Study designs), we formed clinical question (Tab. 2).

Manual searching of retrieved studies was performed not to miss matching studies. A three-step analysis of found articles based on title, abstract, and entire text was undertaken. Two independent reviewers conducted the analysis (PM \& MM) that the third author consulted in case of disagreement (HPS).

The summary of articles analyzed is presented on the flow diagram below (Fig. 1).
Table 1. Inclusion and exclusion criteria of articles

\begin{tabular}{ll}
\hline \multicolumn{1}{c}{ Inclusion Criteria } & \multicolumn{1}{c}{ Exclusion Criteria } \\
\hline - experimental study, cohort study, & - review, case report. \\
clinical trial, multicentre study. & - studies other than on animals and \\
- $\begin{array}{l}\text { available as full text. } \\
\text { spanning the last 10 years of }\end{array}$ & \\
$\begin{array}{l}\text { research. } \\
\text { studies on animals and humans. }\end{array}$ & \\
\hline
\end{tabular}

Table 2. Population Intervention Comparison Outcome Study Design (PICOS) strategy

\begin{tabular}{ll}
\hline Parameter & Description \\
\hline Participants & $\begin{array}{l}\text { animals or humans with neurological disorders - primary brain } \\
\text { tumours, epilepsy, and other neurological disorders. }\end{array}$ \\
\hline Intervention & ketogenic diet intake. \\
\hline Comparison & $\begin{array}{l}\text { healthy controls with no ketogenic supplementation, on normal } \\
\text { diet OR no intervention. }\end{array}$ \\
\hline Outcomes & $\begin{array}{l}\text { changes of cerebral metabolites' concentrations reported as } \\
\text { monitored by magnetic resonance spectroscopy; no other } \\
\text { restrictions were placed on outcome measures. }\end{array}$ \\
\hline Study design & clinical trials or cohort studies or experimental animal studies. \\
\hline
\end{tabular}

A total of 56 records were screened after the implementation of inclusion criteria. Of these, 16 full-text articles were excluded due to poor quality of data concerning no data on the significance of the outcome, manual data entry errors, or data duplications which affected only preclinical studies. After assessment for eligibility, 13 studies were included in the qualitative synthesis.

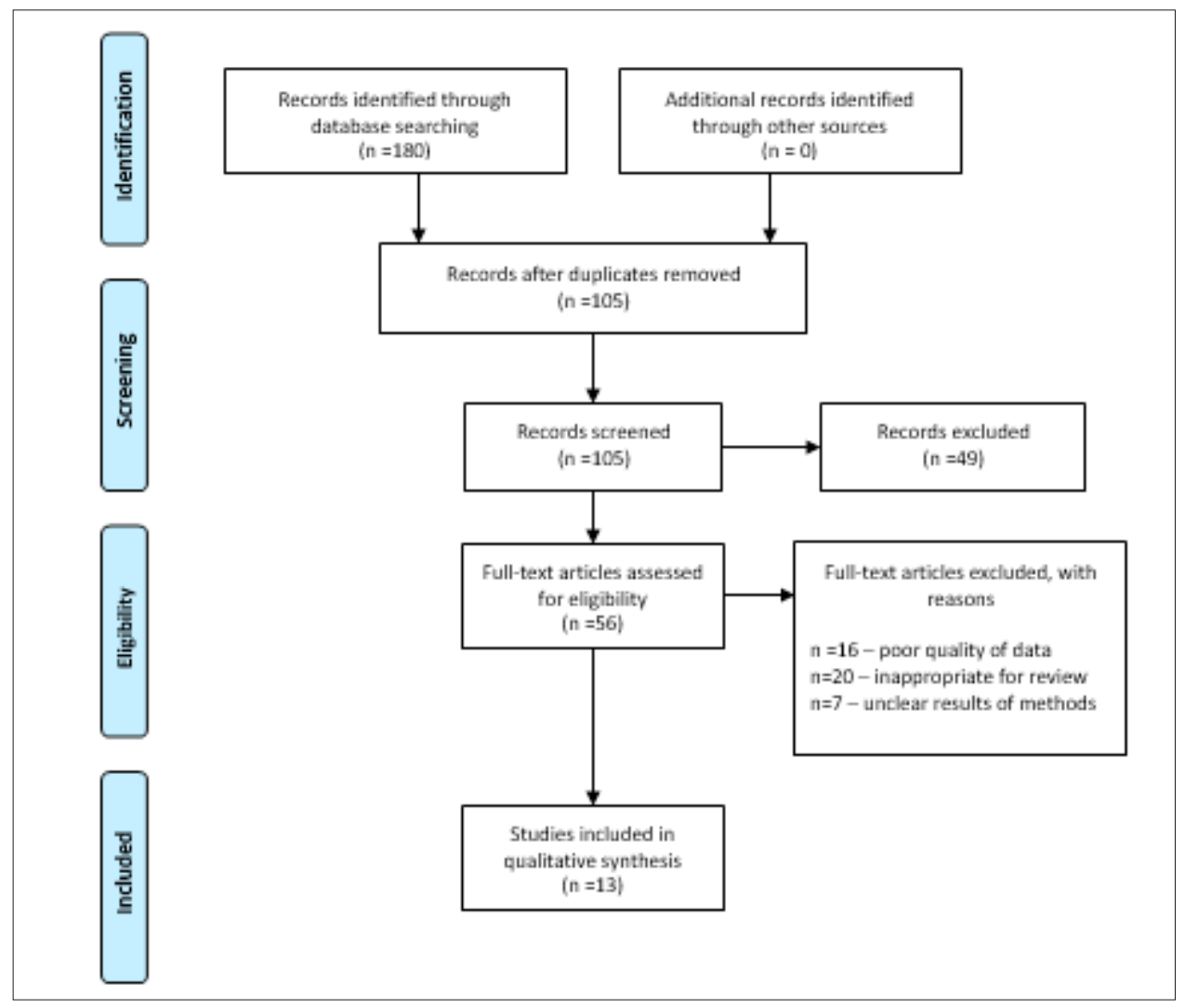

Figure 1. Flow diagram of study identification, inclusion and exclusion criteria 
Table 3. Summary of effects of KD on cerebral metabolic shifts [17 - 26].

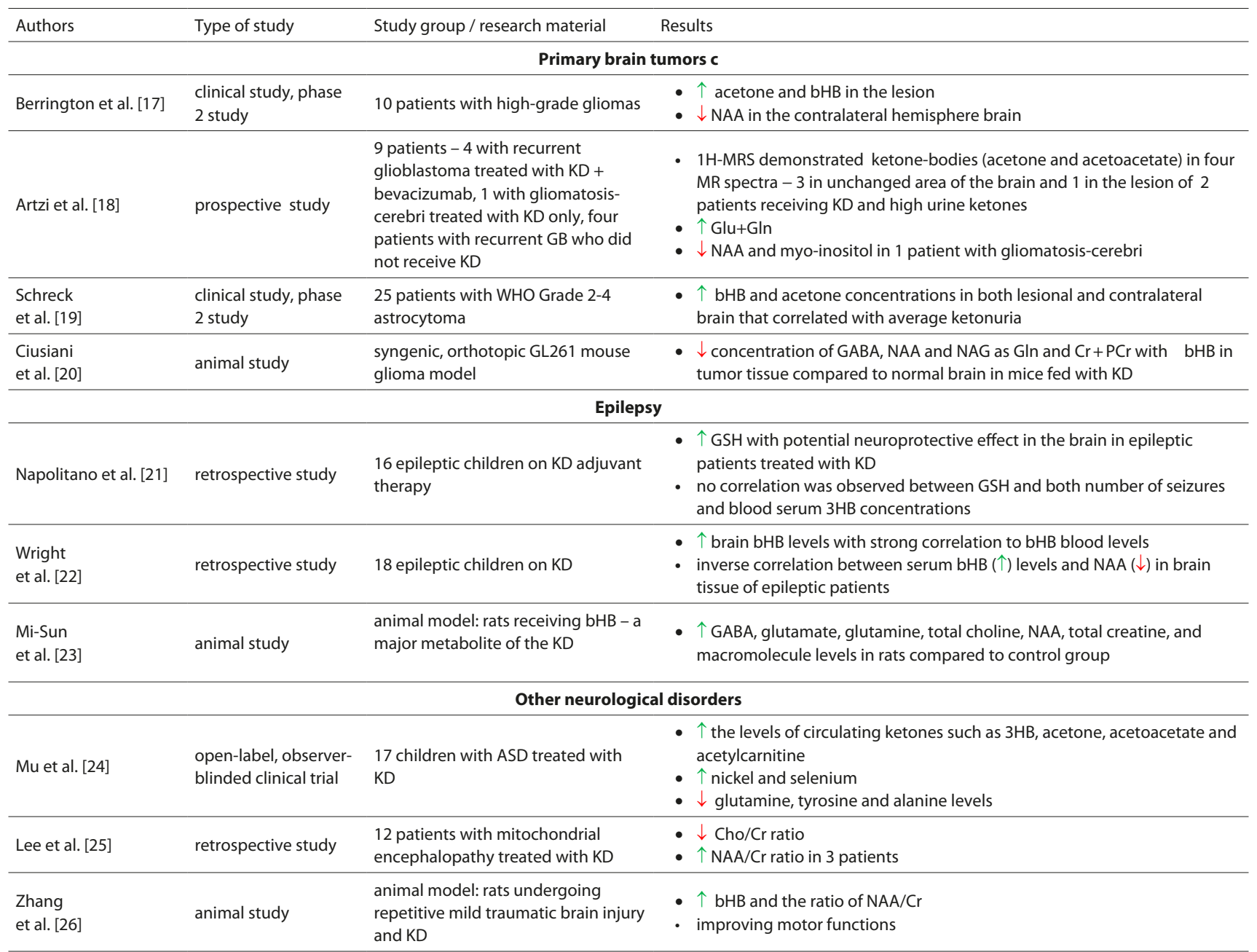

\section{DESCRIPTION OF THE STATE OF KNOWLEDGE}

Impact of ketogenic diet on normal brain function. To assess the usefulness of MRS in monitoring the KD as adjuvant therapy in patients with neurological disorders, it seems justified to define how the KD affects the normal brain [8]. MRS techniques enable non-invasive measurements of neurochemicals in a single voxel or multiple voxels in vivo [14]. Nevertheless, it is worth underlining when assessing neurochemical, metabolic changes of the brain in normal development using MRS, the heterogeneity of the brain needs to be considered because various brain regions present heterogeneous distributions of neurochemical concentrations [15].

Gzieło et al. noticed in her study using MRS performed on a 9.4 $\mathrm{T}$ animal scanner that in young adult rats treated with 4-month-lasting KD, significant increases in concentrations of glutamate (Glu), glutamine (Gln), glutamate + glutamine $(\mathrm{Glu}+\mathrm{Gln})$ and glutathione $(\mathrm{GSH})$ were observed in the cerebral cortex, while the remaining were stable concentrations of $\gamma$-aminobutyric acid (GABA), glucose, glycerophosphocholine, myo-Inositol, N-acetylaspartate (NAA), N-acetylaspartylglutamate (NAAG), phosphocholine, phosphocreatine, taurine [8]. In hippocampal formation, significant increases of Gln, Glu, Glu + Gln, GSH (identical as in cerebral cortex), as well as NAA and NAAG, were revealed. An elevated level of tNAA (NAA + NAAG), being a marker of neuronal integrity and measured by ${ }^{1} \mathrm{H}$ MRS, was also observed in a study by Setkowicz et al. conducted on 25 male Wistar rats on a high fat diet, which correlated well with better memory function [16].

\section{CEREBRAL METABOLISM SHIFTS IN MRS IN PATIENTS WITH NEUROLOGICAL DISORDERS ON A KETOGENIC DIET}

Primary brain tumours. The KD is being extensively studied for its effects on the treatment of neurological disorders such as primary brain tumours, but its physiological effects are not fully understood. This is the reason why some studies attempt to elucidate the link between KD and particular changes in cerebral metabolism.

In a clinical trial by Berrington et al., patients with highgrade gliomas were placed on a modified Atkins diet for 8 weeks, and the possibilities of 3T MRS for controlling the level of brain ketones in the lesion, as well as the contralateral hemisphere, were studied. Pre-post KD data analysis was conducted within a group of 10 patients using the LCM system. The result revealed acetone $(0.06 \pm 0.03 \geq 0.27 \pm 0.06$ IU; $\mathrm{P}=.005)$ and $\beta$-hydroxybutyrate $(\mathrm{bHB})(0.07 \pm 0.07 \geq 0.79$ $\pm 0.32 \mathrm{IU} ; \mathrm{P}=.046)$ increased level in the lesion by week 8 
[17]. At the same time, a significant increase of acetone was noticed in the contralateral brain. Moreover, Berrington's study showed a slight, but also significant, decrease in NAA in the contralateral hemisphere after 8 weeks [17].

In another study by Artzi et al., proton MRS ( $\left.{ }^{1} \mathrm{H}-\mathrm{MRS}\right)$ was performed to assess changes in cerebral metabolism during KD in patients with primary brain tumours. Point-resolved spectroscopy (PRESS) sequence allowed the performance of single voxel spectroscopy used in ${ }^{1} \mathrm{H}$-MRS. Among the group of 9 patients, 4 with recurrent glioblastoma (GB) were treated with $\mathrm{KD}$ in combination with bevacizumab, 1 patient with gliomatosis-cerebri was only on $\mathrm{KD}$ diet, while the remaining 4 patients with recurrent GB did not receive KD. MR scans consisted of conventional imaging and ${ }^{1} \mathrm{H}-\mathrm{MRS}$ acquired from normal appearing-white-matter (NAWM) and particular lesion. In 1 patient with gliomatosis-cerebri who followed the diet for 3 years, an increase in Glu+Gln and decrease in NAA and myo-inositol were noticed as a result of KD [18]. Furthermore, a satisfactory response to the diet was observed in 2 patients in whom ketone bodies, acetone and acetoacetate were detected in $4 \mathrm{MR}$ spectra-three within the NAWM, and 1 concerning the lesion area - respectively, 4 and 25 months after diet initiation [18].

Biological activity of KD was evaluated in a study by Schreck et al. [19] in which 25 patients with WHO Grade 2-4 astrocytoma with stable disease following adjuvant chemotherapy, participated in a GLioma Atkins-based Diet (GLAD) for 8 weeks. This diet is based on 2 days of fasting (calories $<20 \%$ calculated estimated needs) and 5 days of modified Atkins diet each week. Cerebral metabolic activity was assessed using MRS at baseline and week 8 . The result obtained was increased ketone concentrations, including $\mathrm{bHB}$ and acetone in both lesional and contralateral brain that correlated with average ketonuria (bHB R $0.52 ; \mathrm{p}=0.05)$ [19].

The next study confirming that MRS provides an opportunity to control putative metabolic shifts in tumour and healthy brain tissue in high grade gliomas in vivo was conducted by Ciusiani et al. A syngenic mouse model was used in order to monitor the effects of KD on the metabolic responses of tumour with the use of 7T MRS. Glioma cells were injected into the caudate nucleus of mice, fed with a KD or standard nourishment [20]. Follow-up at day 18 after initiating the diet indicated significantly higher plasmatic levels of ketone bodies in mice on $\mathrm{KD}$, compared to mice fed with standard diet. Besides, longer survival was also noticed in the group of mice on KD. MRS revealed differences in metabolic demand between tumour tissue and contralateral normal brain manifesting as a decreased concentration of GABA, NAA and NAG at days 9 and 23 in tumour tissue, compared to normal brain. Furthermore, what was found in MRS was a simultaneous decrease of $\mathrm{Gln}$ and $\mathrm{Cr}+\mathrm{PCr}$, whereas a huge increase in bHB was observed in tumour tissue in comparison to normal brain tissue at day 23 [20]. In contrast, there were no significant metabolic shifts in mice fed with standard diet, including no bHB peak in brain tumour nor in contralateral normal brain. [20]. As was highlighted in a study by Ciusani, the accumulation of bHB in the tumour tissue in mice on KD may be concerned with its increased uptake/release by tumour cells, or their difficulties to use it for mitochondrial metabolism.

Epilepsy. MRS may be a precious tool in monitoring response to ketogenic diets used in the first-line treatment of drug- resistant epilepsy [21, 22]. As has been noticed, epileptic seizures lead to a depletion of mitochondrial GSH, which confirms the correlation between epilepsy and oxidative stress and makes it necessary to monitor GHS level during a KD [21].

In a study by Napolitano et al., estimation of the brain GSH levels in patients with epilepsy on KD adjuvant therapy was conducted by MRS in order to clarify whether the GSH increases are significant in comparison to the control group. For this purpose, MRS evaluated 16 patients on KD and 7 agematched healthy controls. MRS were acquired on a 3T scanner with a 32-channel head-coil. Voxels were set in the gangliocapsular region of the right hemisphere. All examinations were conducted under anesthesia. Additionally, water suppression was also applied to avoid baseline distortion. The results revealed that in patients on $\mathrm{KD}$, higher levels of brain GSH were noticed compared to the control group (KD: $2.5 \pm 0.5 \mathrm{mM}$, HC: $2.0 \pm 0.5 \mathrm{mM})$. Furthermore, there was no link between GSH and the number of epileptic seizures [21].

A study by Wright et al., presented MRS as an effective tool for the non-invasive monitoring of ketosis in paediatric patients with epilepsy is. The aim of the study was to assess the utility of proton MR spectroscopy for detection of bHB in children with epilepsy on $\mathrm{KD}$, and comparing brain parenchymal metabolite ratios with bHB serum concentrations. MR spectroscopy data amounting to 23 spectra from 18 patients were acquired on a $3 \mathrm{~T}$ Trio magnet with a multivoxel point-resolved spectroscopy sequence acquisition set on the basal ganglia. Each patient was under anesthesia during the examination [22]. Results were presented as a ratio of tissue bHB concentration to typically measured cerebral metabolite concentrations, such as NAA, cholinecontaining compounds (Cho), creatine/phosphocreatine (Cr). It was noticed that magnetic resonance spectroscopy revealed measurable bHB with strong correlation to bHB blood levels. In addition, it was demonstrated that an inverse correlation between serum bHB levels and NAA in brain tissue confirmed the anti-epileptogenic properties of KD [22].

In an experimental study, Mi-Sun et al. investigated whether one of the metabolites of $\mathrm{KD}$ - bHB- is effective in infantile spasms (epileptic encephalopathy) in a proposed animal model. Betamethasone was administered to pregnant rats on gestational day 15 . Subsequently, the rat's offspring received a single or prolonged, 3-per-day dose of i.p. bHB., on postnatal day 15 and on postnatal days 12, 13 and 15, respectively. A single dose was administered to rats $30 \mathrm{~min}$ prior NMDA-triggered spasms. Additionally, repeated bouts of spasms on postnatal days 12,13 and 15, with randomized prolonged bHB treatment started as the first spasms occurred [23]. bHB treatment was monitored using ${ }^{1} \mathrm{H}$ MR imaging on a $9.4 \mathrm{~T}$ MR system which revealed at $24 \mathrm{~h}$ after the cluster of spasms, significant $(\mathrm{p}<0.05)$ attenuation of elevated after spasms GABA, NAA, glutamine, glutamate, total creatine, macromolecule-plus lipids levels due to randomized bHB treatment [23]. These cerebral shifts confirmed the satisfactory response to bHB that suppresses the development of spasms.

Other neurological disorders. Chunlong et al. attempted to determine whether shifts in metabolic profiles related to behavioural outcomes in Autism Spectrum Disorders (ASD) in response to $\mathrm{KD}$ treatment. The cohort consisted of 10 typically developed controls (TC) and 17 children with ASD being monitored at baseline for 3 months duringa $\mathrm{KD}$ regimen 
[24]. A multiplatform approach was implemented including gas chromatography-mass spectrometry, ${ }^{1} \mathrm{H}$ nuclear MRS, and inductively coupled plasma-mass spectrometry. In comparison to baseline measurement, $\mathrm{KD}$ increasedthe concentration of ketones, such as 3-hydroxybutyrate $(3 \mathrm{HB})$ and acetone, as well as acetyl carnitine, while reducing glutamine and alanine levels $(\mathrm{p}<0.05)$ [24].

In the next study by Lee et al., the usefulness of MRS in treatment monitoring of mitochondrial encephalopathy (ME) was evaluated. Data acquired using proton MRS was retrospectively assessed in 12 patients with $\mathrm{ME}(\mathrm{M}: \mathrm{F}=7$ : 5 ; mean age $=4.8$ years). All subjects received MRS twice initially and after treatment combined with mitochondrial disease treatment cocktail. Localized ${ }^{1} \mathrm{H}$-MRS was performed on a 3.0T MRI system with use of the 8-channel SENSE head coil [25]. The following parameters were taken into account: choline/creatine ratio, $\mathrm{N}$-acetylaspartate/ creatine ratio, and lactate peak in basal ganglia at $1.2 \mathrm{ppm}$. As a result, in 4 patients, lactate peak was detected. However, disappearance of this peak occurred in 2 patients on follow-up. Furthermore, a significant decrease in choline/creatine ratio was observed on MRS follow-up ( $\mathrm{p}=0.0058$, paired t-test, 2 -tailed). These changes correlated well with the improvement of clinical symptoms, underlining the utility of MRS for monitoring disease progression or improvement in ME [25].

Changes of neurochemical metabolites after KD therapy were also assessed in an experimental study by Zhang et al. on rats undergoing repetitive mild traumatic brain injury. In this animal model, subjects were divided into 3 groups: sham, control, and KD. The last 2 were given 3 rmTBI by a fluid percussion traumatic brain injury device, 24 hours apart [26]. The shifts of neurochemical metabolites were monitored using an MRS that evaluated $\mathrm{N}$-acetylaspartate (NAA)/creatine $(\mathrm{Cr})$, choline compound (Cho)/Cr and $\mathrm{N}$-acetylaspartate/choline ratios. As a result, MRS showed that rmTBI had no effect on the ratios of Cho/Cr and NAA/ Cho. Nevertheless, it reduced the ratio of NAA/Cr which consequently increased after $\mathrm{KD}$, suggesting a potential role of $\mathrm{KD}$ in preventing neurodegeneration [26].

Sensitivity and specificity of MRS in monitoring ketogenic diet. MRS is a more sensitive method than MR imaging in the detection in vivo of shifts of cerebral metabolites. However, the sensitivity and specificity of MRS used in monitoring KD therapy of neurological diseases has not been described exactly. A study by Berrington et al., reported alterations of acetone, acetoacetate and bHB using MRS, but the sensitivity of this method was assessed as being too low due to discrepancies in the detectability of these substances [17]. Acetone was considered as an easily detected compound in contrast to bHB identification. However, sensitivity of this method can be improved by optimized long-TE sequence. It is supposed that performing TE in wider spectra in ms would be more useful in the detection of ketones [22]. However, further research concerning short-echo time should be performed. The diversity and non-specificity of longitudinal patterns of detected metabolites was noted in a study by Artzi, but it concerned only a small sample size and the lack of full compliance by some patients [18]. Slight changes in cerebral metabolism may be non-specific in the MRS imaging because they are not affected solely by the ketogenic diet. Notwithstanding this fact, a significant increase / decrease in the level of some metabolites during KD may be a good indicator for disease monitoring or treatment assessment, which underlines the applicability of MRS.

\section{SUMMARY}

MR spectroscopy is a feasible tool for detecting cerebral metabolic shifts linked to a ketogenic diet. Its utility enables assessment of the effectiveness of the diet therapy used in some neurological disorders by quantification of metabolites in particular cerebral regions. The sensitivity and specificity of MRS may be greater by optimizing the examination techniques, and moreover, by better understanding of the effects of KD on brain metabolism. Nevertheless, further research is needed to determine precisely the applicability of magnetic resonance spectroscopy (MRS) in evaluating the effect of $\mathrm{KD}$, not only experimentally but also in a greater number of human studies, which would guarantee optimal management of patients on an adjuvant high-fat diet due to the presence of certain neurological disorders.

\section{REFERENCES}

1. Murphy EA, Jenkins TJ. A ketogenic diet for reducing obesity and maintaining capacity for physical activity: hype or hope? Curr Opin Clin Nutr Metab Care. 2019 Jul; 22(4): 314-319. https://doi.org/ 10.1097/ MCO.0000000000000572

2. Moreno B, Crujeiras AB, Bellido D, et al. Obesity treatment by very low-calorie-ketogenic diet at two years: reduction in visceral fat and on the burden of disease. Endocrine. 2016; 54(3): 681-690. https://doi. org/10.1007/s12020-016-1050-2

3. Bruci A, Tuccinardi D, Tozzi R, et al. Very Low-Calorie Ketogenic Diet: A Safe and Effective Tool for Weight Loss in Patients With Obesity and Mild Kidney Failure. Nutrients. 2020; 12(2): 333. Published 2020 Jan 27. https://doi.org/10.3390/nu12020333

4. Lambrechts DA, de Kinderen RJ, Vles JS, et al. A randomized controlled trial of the ketogenic diet in refractory childhood epilepsy. Acta Neurol Scand. 2017; 135(2): 231-239. https://doi.org/10.1111/ane.12592

5. Baby N, Vinayan KP, Pavithran N, et al. A pragmatic study on efficacy, tolerability and long term acceptance of ketogenic diet therapy in 74 South Indian children with pharmacoresistant epilepsy. Seizure. 2018; 58: 41-46. https://doi.org/10.1016/j.seizure.2018.03.020

6. Ashrafi MR, Hosseini SA, Zamani GR, et al. The efficacy of the ketogenic diet in infants and young children with refractory epilepsies using a formula-based powder. Acta Neurol Belg. 2017; 117(1): 175-182. https:// doi.org/10.1007/s13760-016-0732-0

7. Kverneland M, Molteberg E, Iversen PO, et al. Effect of modified Atkins diet in adults with drug-resistant focal epilepsy: A randomized clinical trial. Epilepsia. 2018; 59(8): 1567-1576. https://doi.org/10.1111/epi.14457

8. Gzieło K, Janeczko K, Węglarz W, et al. MRI spectroscopic and tractography studies indicate consequences of long-term ketogenic diet. Brain Struct Funct. 2020; 225(7): 2077-2089. https://doi.org/10.1007/ s00429020-02111-9

9. Voss M, Wagner M, von Mettenheim N, et al. ERGO2: A Prospective, Randomized Trial of Calorie-Restricted Ketogenic Diet and Fasting in Addition to Reirradiation for Malignant Glioma. Int J Radiat Oncol Biol Phys. 2020; 108(4): 987-995. https.//doi.org/10.1016/j.ijrobp.2020.06.021

10. Cohen CW, Fontaine KR, Arend RC, et al. Favorable Effects of a Ketogenic Diet on Physical Function, Perceived Energy, and Food Cravings in Women with Ovarian or Endometrial Cancer: A Randomized, Controlled Trial. Nutrients. 2018; 10(9): 1187. Published 2018 Aug 30. https://doi.org/10.3390/nu10091187

11. Cohen CW, Fontaine KR, Arend RC, et al. A Ketogenic Diet Reduces Central Obesity and Serum Insulin in Women with Ovarian or Endometrial Cancer. J Nutr. 2018; 148(8): 1253-1260. https://doi. org/10.1093/jn/nxy119

12. Khodabakhshi A, Akbari ME, Mirzaei HR, et al. Feasibility, Safety, and Beneficial Effects of MCT-Based Ketogenic Diet for Breast Cancer Treatment: A Randomized Controlled Trial Study. Nutr Cancer. 2020; 72(4): 627-634. https://doi.org/10.1080/01635581.2019.1650942 
13. Zahra A, Fath MA, Opat E, et al. Consuming a Ketogenic Diet while Receiving Radiation and Chemotherapy for Locally Advanced Lung Cancer and Pancreatic Cancer: The University of Iowa Experience of Two Phase 1 Clinical Trials. Radiat Res. 2017; 187(6): 743-754. https:// doi.org/10.1667/RR14668.1

14. Lee P, Adany P, Choi IY. Imaging based magnetic resonance spectroscopy (MRS) localization for quantitative neurochemical analysis and cerebral metabolism studies. Anal Biochem. 2017; 529: 40-47. https://doi.org/10.1016/j.ab.2017.01.007

15. Emir UE, Auerbach EJ, Van De Moortele PF, et al. Regional neurochemical profiles in the human brain measured by ${ }^{1} \mathrm{H}$ MRS at 7 T using local $B_{1}$ shimming. NMR Biomed. 2012; 25(1): 152-160. https:// doi.org/10.1002/nbm.1727

16. Setkowicz Z, Gaździńska A, Osoba JJ, et al. Does Long-Term High Fat Diet Always Lead to Smaller Hippocampi Volumes, Metabolite Concentrations, and Worse Learning and Memory? A Magnetic Resonance and Behavioral Study in Wistar Rats. PLoS One. 2015; 10(10): e0139987. Published 2015 Oct 8. https://doi.org/10.1371/journal. pone. 0139987

17. Berrington A, Schreck KC, Barron BJ, et al. Cerebral Ketones Detected by 3 T MR Spectroscopy in Patients with High-Grade Glioma on an Atkins-Based Diet. AJNR Am J Neuroradiol. 2019; 40(11): 1908-1915. https://doi.org/10.3174/ajnr.A6287

18. Artzi M, Liberman G, Vaisman N, et al. Changes in cerebral metabolism during ketogenic diet in patients with primary brain tumors: $1 \mathrm{H}-\mathrm{MRS}$ study. J Neurooncol. 2017; 132(2): 267-275. https.//doi.org/10.1007/ s11060-016-2364-x

19. Schreck KC, Hsu FC, Berrington A, et al. Feasibility and Biological Activity of a Ketogenic/Intermittent-Fasting Diet in Patients With Glioma [published online ahead of print, 2021 Jul 7]. Neurology.
2021; 10.1212/WNL.0000000000012386. https://doi.org/10.1212/ WNL.0000000000012386

20. Ciusani E, Vasco C, Rizzo A, et al. MR-Spectroscopy and Survival in Mice with High Grade Glioma Undergoing Unrestricted Ketogenic Diet [published online ahead of print, 2020 Sep 21]. Nutr Cancer. 2020; 1-8. https://doi.org/10.1080/01635581.2020.1822423

21. Napolitano A, Longo D, Lucignani M, et al. The Ketogenic Diet Increases In Vivo Glutathione Levels in Patients with Epilepsy. Metabolites. 2020; 10(12): 504. Published 2020 Dec 10. https.//doi.org/10.3390/ metabo10120504

22. Wright JN, Saneto RP, Friedman SD. $\beta$-Hydroxybutyrate Detection with Proton MR Spectroscopy in Children with Drug-Resistant Epilepsy on the Ketogenic Diet. AJNR Am J Neuroradiol. 2018; 39(7): 1336-1340. https.//doi.org/10.3174/ajnr.A5648

23. Yum MS, Lee M, Woo DC, et al. $\beta$-Hydroxybutyrate attenuates NMDAinduced spasms in rats with evidence of neuronal stabilization on MR spectroscopy. Epilepsy Res. 2015; 117: 125-132. https.//doi.org/10.1016/j. eplepsyres.2015.08.005

24. Mu C, Corley MJ, Lee RWY, et al. Metabolic Framework for the Improvement of Autism Spectrum Disorders by a Modified Ketogenic Diet: A Pilot Study. J Proteome Res. 2020; 19(1): 382-390. https.//doi. org/10.1021/acs.jproteome.9b00581

25. Lee SK, Kim J, Kim HD, et al. Initial experiences with proton MR spectroscopy in treatment monitoring of mitochondrial encephalopathy. Yonsei Med J. 2011; 51(5): 672-675. https.//doi. org/10.3349/ymj.2010.51.5.672

26. Zhang F, Wu H, Jin Y, et al. Proton Magnetic Resonance Spectroscopy (H1-MRS) Study of the Ketogenic Diet on Repetitive Mild Traumatic Brain Injury in Adolescent Rats and Its Effect on Neurodegeneration. World Neurosurg. 2018; 120: e1193-e1202. https.//doi.org/10.1016/j. wneu.2018.09.037 